\title{
Getting Warmer
}

The approach to clinical conundrums by an expert clinician is revealed through the presentation of an actual patient's case in an approach typical of a morning report. Similarly to patient care, sequential pieces of information are provided to the clinician, who is unfamiliar with the case. The focus is on the thought processes of both the clinical team caring for the patient and the discussant.

This icon represents the patient's case. Each paragraph that follows represents the discussant's thoughts.

Shaili Rajput, MD, MPH ${ }^{1 \star}$, John Schmidt, MD², Jennifer Stojan, MD², Callistus Ditah, MD³, Nathan Houchens, MD

${ }^{1}$ Department of Pediatrics, University of Michigan, Ann Arbor, Michigan; ${ }^{2}$ Departments of Internal Medicine and Pediatrics, University of Michigan, Ann Arbor, Michigan; ${ }^{3}$ General Surgery Residency Program, University of Wisconsin, Madison, Wisconsin; ${ }^{4}$ Department of Internal Medicine, University of Michigan and Veterans Affairs Ann Arbor Healthcare System, Ann Arbor, Michigan.

A 3-month-old otherwise healthy, immunized female presented to clinic with 2 days of intermittent lowgrade fevers (maximum, $100^{\circ} \mathrm{F}$ ), decreased oral intake, and sleepiness. Her pediatrician noted a faint, maculopapular rash on her trunk and extremities with mild conjunctival injection bilaterally that appeared that day, according to her mother. The infant otherwise appeared alert, well-hydrated, and without respiratory distress. She had no history of sick contacts or recent travel. She was prescribed amoxicillin for empiric treatment of a possible bacterial sinusitis or pharyngitis, despite a negative rapid strep antigen test.

At this age, multiple conditions can cause rashes. Given that this is early in the course of illness, without focal symptoms but with low-grade fevers, the initial differential diagnosis is broad and would include infectious, rheumatologic, and hematologic-oncologic etiologies, although the latter would be less likely. While the patient's mother reports decreased oral intake, the fact that the patient is alert and appears hydrated is encouraging, suggesting time to observe and see if other symptoms present that may assist in elucidating the cause. The history of increased sleepiness warrants further investigation of meningeal signs, which would point to a central nervous system infection.

While streptococcal infection is possible, it would be uncommon at this age. The patient would have a higher fever and focal infection, and the rash does not appear consistent unless it was described as "sandpaper" in feel and appearance. A negative rapid strep test, while not sensitive, further supports this impression. A low-grade fever and rash would be consistent with a viral syndrome and, given the conjunctival injection, adenovirus, cytomegalovirus, rhinovirus, and Epstein Barr virus (EBV) are possibilities. Without ocular

*Address for Correspondence and Reprint Requests: Shaili Rajput, MD, MPH, 1885 Bay Road, East Palo Alto, CA 94301; Telephone: 650-383-2342; Fax: 650-321-1610; Email: shaili.rajput@gmail.com

Received: April 1, 2016; Revised: June 24, 2016; Accepted: June 28, 2016. 2017 Society of Hospital Medicine DOI 10.1002./jhm.2675 discharge, bacterial conjunctivitis would be unlikely. Another consideration would be Kawasaki disease, though it would be too early to diagnose this condition since at least 5 days of fever are required. Next steps include a detailed physical examination, looking for other focal signs such as swelling or desquamation of hands and feet, lymphadenopathy, strawberry tongue, and mucositis. Rather than empirically starting antibiotics, it would be more reasonable to observe her with close outpatient follow-up. The patient's family should be instructed to monitor for additional and/or worsening symptoms, further decreased oral intake, signs of dehydration, or changes in alertness.

At home, the patient completed 5 doses of amoxicillin but continued to be febrile (maximum, $102.6^{\circ} \mathrm{F}$ ). She was taken to a local emergency department on day 6 of her illness. She had worsening conjunctival injection and progression of the rash, involving the palms and soles. She was noted to have edema of hands and feet without desquamation (Figure 1). She had no oral mucous membrane changes and no cervical lymphadenopathy. Cerebrospinal fluid (CSF) was unremarkable, and empiric treatment with intravenous (IV) ceftriaxone was initiated. Complete blood count was notable for a white blood cell (WBC) count of $18.9 \mathrm{k} / \mu \mathrm{L}$ (normal range, 6.0-17.0); hemoglobin, $7.6 \mathrm{~g} / \mathrm{dL}$ (normal range, 10-13); mean corpuscular volume, 84 (normal range, 74-108); and platelet count, 105 $\mathrm{k} / \mathrm{\mu L}$ (normal range, 150-400). A peripheral blood smear revealed no abnormal cells. C-reactive protein (CRP) was

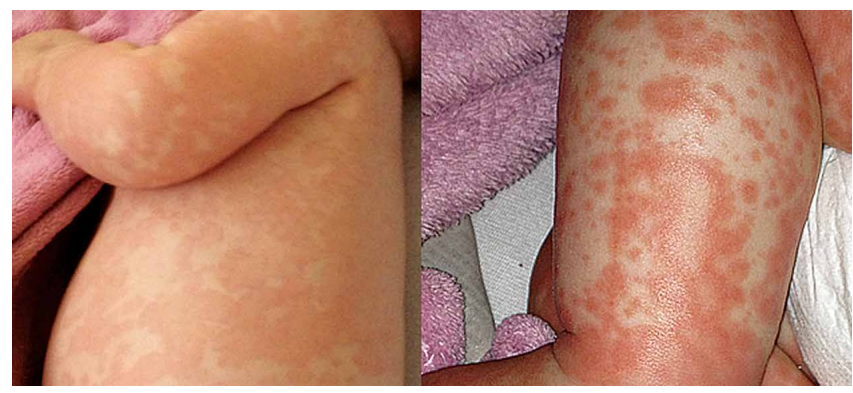

FIG. 1. Fine, erythematous, blanching maculopapular rash involving the palms and the soles with associated edema. 
elevated at $6.5 \mathrm{mg} / \mathrm{dL}$ (normal range, 0.0-0.6). She was admitted for further management.

Infection remains on the differential diagnosis given the elevated WBC count. Since the patient has completed a reasonable course of antibiotics, a bacterial infection would be less likely but not fully excluded. The cultures obtained would be helpful if they become positive, but given that the patient has been on antibiotics, a negative culture may represent partial sterilization and would not rule out infection. A viral infection continues to be high on the differential, but one would expect that symptoms and fever would have begun to abate. The normal peripheral blood smear makes a hematologic disorder less likely.

Kawasaki disease has risen on the differential with 5 days of fever surpassing $102^{\circ} \mathrm{F}$. She has 3 of 5 primary clinical criteria, including conjunctival injection, rash, and edema of the hands and feet. Desquamation of the peripheral extremities would not be expected until the convalescent phase. A diagnosis of typical Kawasaki disease would require a fourth criterion, either oral mucous membrane changes or cervical lymphadenopathy. She meets the criteria for atypical or incomplete Kawasaki disease, which requires only fever for at least 5 days, elevated CRP, and 2 or 3 additional clinical criteria. She also meets supplemental laboratory criteria with an elevated WBC count greater than $15,000 / \mu \mathrm{L}$, normocytic and normochromic anemia for age, and elevated CRP. Urinalysis positive for pyuria or serum albumin less than $3 \mathrm{~g} / \mathrm{dL}$ would lend further support but is not necessary. Fever of 7 or more days in a child less than 6 months old without other explanation would also increase the likelihood of incomplete Kawasaki disease. Admission to the hospital, treatment with IV immunoglobulin (IVIg), and echocardiography to evaluate for typical cardiac involvement (eg, aneurysms, coronary arteritis, and pericardial effusion) are the appropriate next steps.

The patient was diagnosed with atypical Kawasaki disease. A transthoracic echocardiogram was normal on admission. On day 7 of her illness, she was treated with 1 dose of IVIg at $2 \mathrm{~g} / \mathrm{kg}$ and high-dose aspirin at $100 \mathrm{mg} /$ $\mathrm{kg}$ per day in divided doses. Despite this treatment, she continued to be febrile and was given a second dose of IVIg on day 9. Her fevers persisted.

In Kawasaki disease, persistent fever is concerning for longterm sequelae, including coronary artery aneurysms. Continued treatment is reasonable. After 2 doses of IVIg with a cumulative dose of $4 \mathrm{~g} / \mathrm{kg}$, it is prudent to switch therapy to IV methylprednisolone $30 \mathrm{mg} / \mathrm{kg}$ with repeated doses as needed for up to 3 days should her fevers persist.

Her blood culture was negative. EBV serology, enterovirus polymerase chain reaction, and viral cultures were negative. Chest radiography on day 9 was normal. Abdominal ultrasonography on day 10 showed hydrops of the gallbladder.
The patient was started on IV corticosteroids on day 11 with resolution of her fevers and improvement in her rash. A repeat echocardiogram revealed new findings of dilated left main, left anterior descending, and right coronary arteries. On day 13, a steroid wean was attempted because she had remained afebrile for more than 48 hours, but the wean was halted due to recurrence of fevers and rash. Her high-dose aspirin was reduced to $81 \mathrm{mg}$ PO daily on day 14 , and she was started on enoxaparin injections.

It is unusual for Kawasaki disease not to respond to 2 doses of IVIg, followed by corticosteroids. As such, the differential diagnosis must be revisited. The findings of coronary artery dilation, prolonged fever, and rash corroborate the diagnosis of Kawasaki disease, although this could be an atypical presentation of another vasculitis. Systemic onset juvenile idiopathic arthritis usually affects children at 2 to 5 years old and is, therefore, less likely. Henoch-Schönlein purpura manifests with a rash but is often associated with diarrhea. There does not appear to be objective evidence of polyarteritis nodosa, although biopsy or angiography would be required to make this diagnosis. Hydrops of the gallbladder is an over-distention of the organ filled with watery or mucoid content. While hydrops can be noninflammatory and seen in gallstone disease, it can also occur in vasculitides. Despite the reassuring serologies, false negative results are possible. Thus, these viral infections are not eliminated, but they are less likely. Given the echocardiogram findings and continued concern for atypical Kawasaki disease, high-dose aspirin should be continued. It is reasonable to consider rheumatology consultation for assessment and recommendations as to length of steroid treatment and/or alternative interventions.

Pediatric cardiology was consulted. Repeat echocardiogram on day 16 showed an increase in the size of her coronary artery aneurysms, and her fevers persisted. Computed tomography scan of the abdomen and pelvis with contrast, obtained to further evaluate for a source of infection, was unremarkable.

The patient was transferred to a tertiary care institution on day 19, at which time she remained on aspirin, enoxaparin, and oral corticosteroids. On arrival, her temperature was $101.3^{\circ} \mathrm{F}$, heart rate 225 beats per minute, and respiratory rate 57 breaths per minute. She was fussy with bilateral conjunctivitis and a maculopapular rash involving palms, soles, and right infraorbital region. Laboratory studies were significant for a WBC count of $30.3 \mathrm{k} /$ $\mu \mathrm{L}$; hemoglobin, $10.9 \mathrm{~g} / \mathrm{dL}$; platelets, $106 \mathrm{k} / \mu \mathrm{L}$; and CRP, $8.3 \mathrm{mg} / \mathrm{dL}$.

Pediatric rheumatology was consulted on day 20. The patient was treated with 3 days IV pulse-dose methylprednisolone at $30 \mathrm{mg} / \mathrm{kg}$ daily. Her fevers resolved, although her CRP level remained elevated. She was treated with 1 dose of infliximab $10 \mathrm{mg} / \mathrm{kg} \mathrm{IV}$ on day 24 , followed by 1 dose of anakinra $15 \mathrm{mg}$ subcutaneously on day 27 due to persistently elevated CRP. 
The symptoms and diagnostic evaluation remain most consistent with atypical Kawasaki disease. Her tachycardia and tachypnea are likely driven by her fever and fussiness, and should be followed closely. The elevated WBC is likely a consequence of the steroids and demargination of neutrophils. The elevated and increasing CRP is a marker of acute inflammation. The adage "treat the patient, not the numbers" comes to mind, because it is reassuring that the patient's overall clinical picture seems to be improving with resolution of her fevers. However, further discussion with the pediatric rheumatology consultant is prudent, specifically regarding the significance of the persistently elevated CRP, refinement of the differential diagnosis including the potential for other vasculitides and appropriate evaluation of such, as well as recommendations for further treatment.

$\triangle$ The patient was noted to have ongoing fevers. Based on reports of success with cyclophosphamide in refractory Kawasaki disease, she was treated with 2 doses at $60 \mathrm{mg}$ IV per dose starting on day 28. Her CRP level decreased. Cardiology and rheumatology consultants recommended magnetic resonance imaging/magnetic resonance angiography of the chest, abdomen, and pelvis with and without contrast. These studies revealed dilation of the axillary and brachial arteries (Figure 2).

The response to cyclophosphamide confirms an autoimmune/inflammatory process. The imaging results and pattern are most consistent with either Kawasaki disease or polyarteritis nodosa. Therefore, rheumatology's input will be

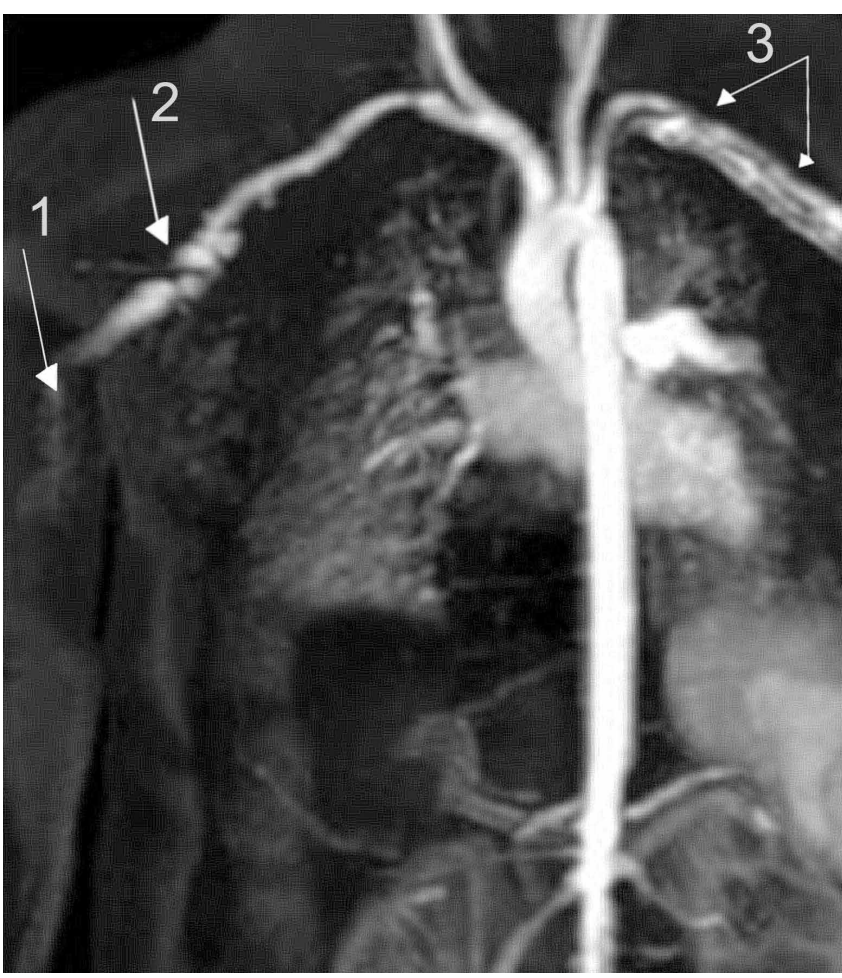

FIG. 2. Tortuous and aneurysmal dilation of the right proximal brachial artery (Arrow 1) and axillary artery (Arrow 2), and probable left axillary dilation (Arrow 3). invaluable with regard to which diagnosis is most likely, additional diagnostic testing, and appropriate medical regimen and follow-up plans.

Systemic extracoronary vascular inflammation on imaging and the refractory nature of the patient's disease process, despite appropriate treatment for Kawasaki disease, led to the diagnosis of childhood polyarteritis nodosa (PAN). The patient was discharged home and closely followed in rheumatology clinic. Her most recent outpatient visit 1 year after the initial onset of her illness showed no further fevers or rashes, normal inflammatory markers, and stabilization of her coronary aneurysms on daily maintenance azathioprine.

\section{DISCUSSION}

Fever with an accompanying rash is a common issue in children. The extensive differential diagnosis includes infectious diseases, rheumatologic disorders, and medication reactions (Table 1). A thorough history and physical examination are essential in guiding the physician toward the proper diagnosis and management. Important information includes patient age, season, associated symptoms, exposure to sick contacts, travel history, host immune status, and immunization history. Fever duration and pattern must be elicited, as should features of the rash, including temporal relationship to the fever, distribution, progression, and morphology. ${ }^{1}$

When unexplained fever persists for 5 days or more in the pediatric patient, the diagnosis of $\mathrm{KD}$ must be suspected. $\mathrm{KD}$ is an acute, febrile, primary systemic vasculitis affecting small- and medium-sized vessels, with a predilection for coronary arteries. ${ }^{2} \mathrm{KD}$ affects younger children, with approximately $85 \%$ of cases occurring in children under 5 years old.

\begin{tabular}{ll}
\hline TABLE 1. Common Causes of Fever with \\
Accompanying & Rash in Children \\
\hline Viral infections & Measles \\
& Rubella \\
& Roseola (human herpesvirus 6 and 7) \\
& Erythema infectiosum (parvovirus) \\
& Epstein-Barr virus \\
& Cytomegalovirus \\
& Other nonspecific viral illnesses \\
\hline Bacterial infections & Scarlet fever \\
& Lyme disease \\
& Rocky Mountain spotted fever \\
\hline Rheumatologic disorders & Acute rheumatic fever \\
& Systemic juvenile idiopathic arthritis \\
& Kawasaki disease \\
& Polyarteritis nodosa \\
& Henoch-Schönlein purpura \\
\hline Medication reactions & Erythema multiforme \\
& Stevens-Johnson syndrome/toxic epidermal necrolysis \\
& Drug reaction with eosinophilia and systemic symptoms (DRESS) \\
& syndrome \\
\hline
\end{tabular}




\begin{tabular}{l}
\hline TABLE 2. Diagnostic Criteria of Kawasaki Disease \\
and Childhood Polyarteritis Nodosa \\
\hline Kawasaki Disease \\
\hline Fever of at least 5 days' duration plus at least 4 of the following: \\
- Polymorphous exanthema \\
- Bilateral conjunctival injection with limbic sparing \\
- Cervical lymphadenopathy $>1.5 \mathrm{~cm}$ in diameter \\
- Lip and oral cavity changes (erythema, cracked lips, strawberry tongue) \\
- Extremity changes (erythema of palms and soles, edema, later desquamation) \\
\hline Childhood Polyarteritis Nodosa \\
\hline Biopsy showing small- and medium-sized artery necrotizing vasculitis or aneurysms \\
or occlusions on angiography plus at least 1 of the following: \\
- Skin involvement (livedo reticularis, nonspecific vasculitis lesions, tender subcutaneous nodules) \\
- Myalgia or muscle tenderness \\
- Mononeuropathy or polyneuropathy \\
- Systemic hypertension \\
- Renal involvement (proteinuria, hematuria, red blood cell casts, or GFR <50\% normal value \\
for age) \\
\hline NOtE: Abbreviation: GFR, glomerular filtration rate. \\
\hline
\end{tabular}

KD has a higher incidence in Asian populations, suggesting a possible genetic predisposition. ${ }^{3}$ The etiology of $\mathrm{KD}$ is not well understood, but infection and immune dysregulation have been proposed as contributing factors. KD is the leading cause of acquired heart disease in developed countries. ${ }^{2}$

The diagnosis of KD is made clinically (Table 2). Atypical $\mathrm{KD}$ is considered in patients with at least 5 days of fever but only 2 or 3 clinical criteria. Supportive laboratory findings include elevated inflammatory markers, anemia, neutrophilia, abnormal plasma lipids, low albumin, sterile pyuria, CSF pleocytosis, and elevated serum transaminases. Two-dimensional echocardiography should be performed in all children with definite or suspected $\mathrm{KD}$ at the time of diagnosis, 1 to 2 weeks later, and 6 weeks following discharge for evaluation of the coronary arteries, left ventricular function, and valve function. The American Heart Association recommends follow-up echocardiography at 1 year in children without coronary vessel involvement. ${ }^{4}$

Treatment is aimed at minimizing inflammation and coronary artery involvement, and should be initiated promptly. ${ }^{5}$ Therapy includes a single infusion of high-dose IVIg and aspirin; ${ }^{6,7}$ the latter is initially provided at high anti-inflammatory doses, followed by lower antithrombotic doses once fever and laboratory markers have resolved. ${ }^{2}$ Aspirin can be discontinued if there is no evidence of coronary involvement at the 6-week follow-up echocardiogram. ${ }^{5} \mathrm{~A}$ second dose of IVIg is given within 48 hours for refractory cases, defined as persistent fever following the first dose of IVIg. ${ }^{4}$ Fifteen percent of children have refractory illness, and refractory KD is associated with a higher risk of coronary artery lesions. ${ }^{5}$ Additional agents that suppress immune activation and cytokine secretion contributing to KD pathogenesis have been studied. Corticosteroids inhibit phospholipase A, an enzyme required for production of inflammatory markers. ${ }^{8}$ Infliximab, a tumor necrosis factor-alpha inhibitor, has been shown to reduce duration of fever and length of hospital stay. ${ }^{8,9}$ Anakinra, an interleukin-1 receptor antagonist, has been shown to decrease fever duration and prevent progression of vascular injury in cases of refractory KD. ${ }^{10}$ There is, however, a lack of sufficient evidence and consensus on best practice..$^{8-10}$

If inflammation, evidenced by fever, elevated inflammatory markers (such as erythrocyte sedimentation rate, CRP), or vessel involvement on imaging, persists or worsens despite standard therapy, physicians should seek alternative diagnoses. This patient's extracoronary vascular inflammation and favorable response only to cyclophosphamide led to the diagnosis of systemic PAN. Like KD, PAN is a multi-system vasculitis affecting small- and medium-sized vessels. Unlike $\mathrm{KD}$, PAN is rarely seen in children. ${ }^{11}$ Historically, PAN was thought to represent an extreme fatal end of the KD spectrum. Today, PAN is accepted as a separate entity. Clinical features and histological findings often overlap with $\mathrm{KD}$, creating a diagnostic dilemma for providers. ${ }^{12}$

At the onset of illness, clinical features of systemic PAN may include recurrent fever, weight loss, and myalgia, with gradual progression to multi-organ system involvement. Laboratory assessment reveals elevated inflammatory markers and leukocytosis. Thrombocytosis, anemia, proteinuria, and hematuria may be present. A positive antineutrophil cytoplasmic antibody is rare in PAN and should raise suspicion for a microscopic polyangiitis, which is distinguished from PAN by small vessel involvement only. When compared to $\mathrm{KD}$, cardiac vessel involvement in PAN is more variable. ${ }^{11}$ Diagnostic criteria for childhood PAN are listed in Table $2 .{ }^{13}$

Treatment of PAN is aimed at inducing remission with high-dose steroids and cyclophosphamide. Maintenance of remission is achieved using low-dose steroids and azathioprine. ${ }^{11}$ Total duration of treatment averages 2 to 3 years, with a minimum of 18 months. ${ }^{14}$ Plasma exchange has been used in severe, life-threatening cases. ${ }^{11}$ Prognosis for children with PAN is more favorable compared to adults with PAN, in whom the mortality rate is as high as $20 \%$ to $30 \%$, even with aggressive treatment. In 1 multicenter study of childhood and adolescent PAN, overall mortality was $1.1 \% .{ }^{15}$

This patient initially presented with findings consistent with KD. As her inflammatory markers remained elevated and fevers persisted, her physicians appropriately reconsidered the etiology of her symptoms, thereby "getting warmer" in the search for the correct diagnosis of systemic PAN, a rare disease and a separate entity from $\mathrm{KD}$. Recognizing the overlapping and distinct clinical features of each entity can promote more timely and appropriate selection of therapy, thereby minimizing clinical manifestations and complications associated with each vasculitis.

\section{KEY TEACHING POINTS}

- KD and childhood PAN are disseminated vasculitides affecting small- and medium-sized vessels. Although they are distinct entities, KD and PAN exhibit overlapping clinical and pathological features that make appropriate 
diagnosis and treatment challenging.

- In cases of refractory KD, alternative diagnoses should be considered.

- Recognizing the individual features of both entities is imperative because treatment differs: KD is treated with high-dose aspirin and IVIg; corticosteroids and immunosuppressive agents are used to treat PAN.

Disclosure: Nothing to report.

\section{References}

1. McKinnon HD Jr, Howard T. Evaluating the febrile patient with a rash. Am Fam Physician. 2000;62:804-816.

2. Dimitriades V, Brown AG, Gedalia A. Kawasaki disease: pathophysiology, clinical manifestations, and management. Curr Rheumatol Rep. 2014;16:423.

3. Callinan L, Holman RC, Vugia DJ, Schonberger LB, Belay ED. Kawasaki disease hospitalization rate among children younger than 5 years of age in California, 2003-2010. Pediatr Infect Dis J. 2014;33:781-783.

4. Newburger JW, Takahashi M, Gerber MA, Gewirtz MH, Tani LY, Burns JC, et al. Committee on Rheumatic Fever, Endocarditis and Kawasaki Disease, Council on Cardiovascular Disease in the Young, American Heart Association; American Academy of Pediatrics. Diagnosis, treatment, and long-term management of Kawasaki disease: a statement for health professionals from the Committee on Rheumat ic Fever, Endocarditis and Kawasaki Disease, Council on Cardiovascular Disease in the Young, American Heart Association. Circulation. 2004;110:2747-2771.
5. Son M, Newburger JW. Kawasaki disease. Pediatr Rev. 2013;34:151-61.

6. Newberger JW, Takahasi M, Beiser AS, et al. A single intravenous infusion of gammaglobulin as compared with four infusions in treatment of acute Kawasaki syndrome. N Engl J Med. 1991;324:1633-1639.

7. Dajani AS, Taubert KA, Gerber MA, et al. Diagnosis and therapy of Kawasaki disease in children. Circulation. 1993;87:1776-1780.

8. Saneeymehri S, Baker K, So TY. Overview of pharmacological treatment options for pediatric patients with refractory Kawasaki disease. J Pediatr Pharmacol Ther. 2015;20:163-177.

9. Brogan R, Eleftheriou D, Gnanapragasam J, Klein NJ, Brogan PA. Infliximab for the treatment of intravenous immunoglobulin resistant Kawasaki disease complicated by coronary artery aneurysms: a case report. Pediatr Rheumatol Online J. 2009;7:3.

10. Cohen S, Tacke CE, Straver B, Meijer N, Kuipers IM, Kuijpers TW. A child with severe relapsing Kawasaki disease rescued by IL-1 receptor blockade and extracorporeal membrane oxygenation. Ann Rheum Dis. 2012;71:2059-2061.

11. Kelly A, Tizard E. Vasculitis in children. Paediatrics and Child Health. 2010;20:6572.

12. Yamazaki-Nakashimada MA, Espinosa-Lopez M, Hernandez-Bautista V, Espinosa-Padilla S, Espinosa-Rosales F. Catastrophic Kawasaki disease or juvenile polyarteritis nodosa? Semin Arthritis Rheum. 2006;35:349-354.

13. Ozen S, Pistorio A, Iusan SM, et al. EULAR/PRINTO/PRES criteria for Henoch-Schönlein purpura, childhood polyarteritis nodosa, childhood Wegener granulomatosis and childhood Takayasu arteritis: Ankara 2008. Part II: Final classification criteria. Ann Rheum Dis. 2010;69:798-806.

14. Eleftheriou D, Brogan PA. Vasculitis in children. Best Pract Res Clin Rheumatol. 2009;23:309-323.

15. Ozen S, Anton J, Arisoy N, et al. Juvenile polyarteritis: results of a multicenter survey of 110 children. J Pediatr. 2004;145:517-522. 